
$\mathrm{CHM}$

\title{
CARD LOOM WEAVING
}

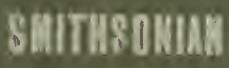

LiBRaBies 
ES SMITHSONIAN INSTITUTION

NYINOSHLIWS S 31 YYQ \& 17
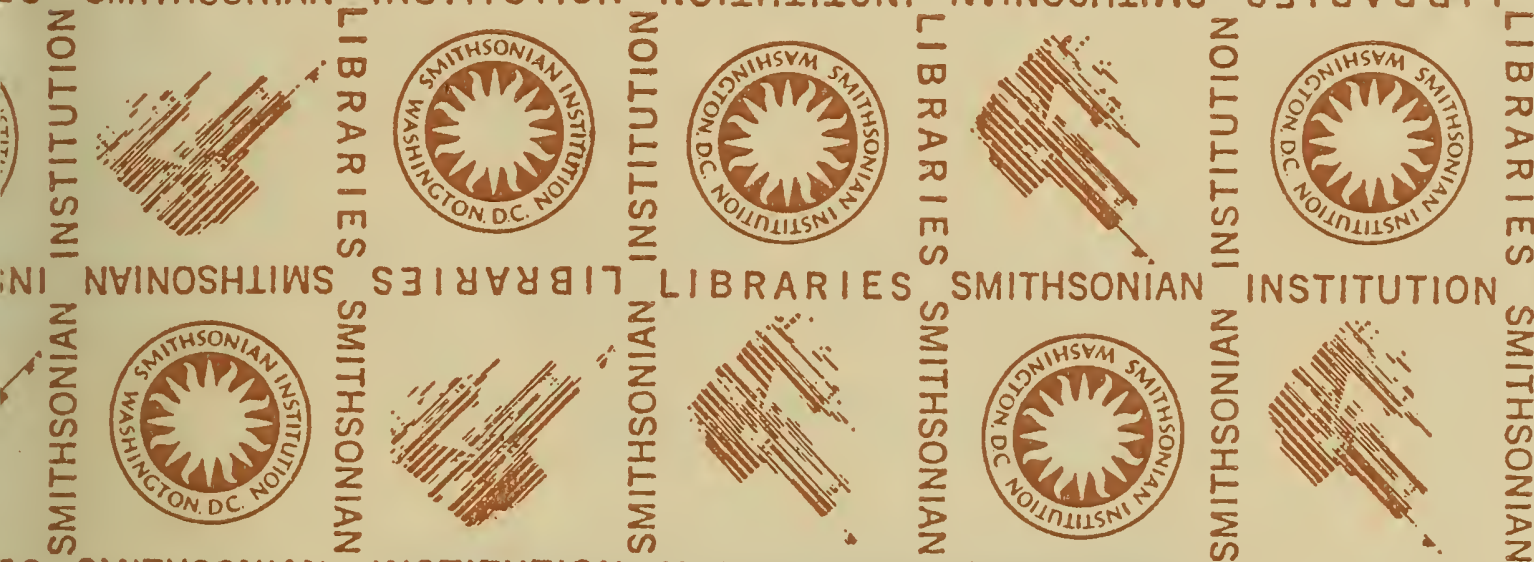

SMITHSONIAN
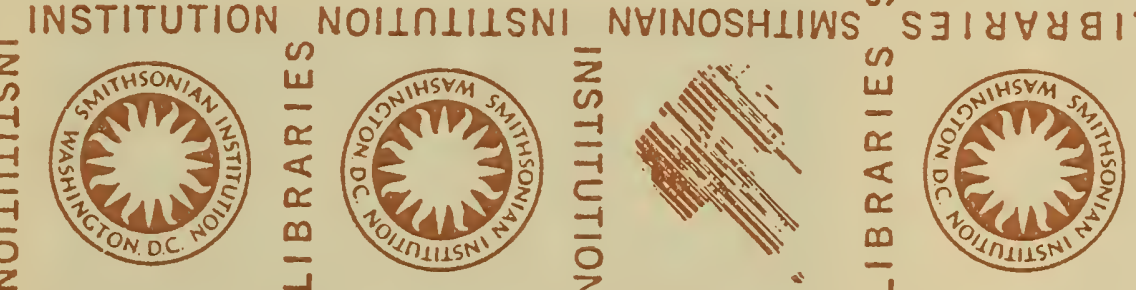

$z$
$=$
$=$
$=$
$\overline{0}$
$z$

NHINOSHIIWS
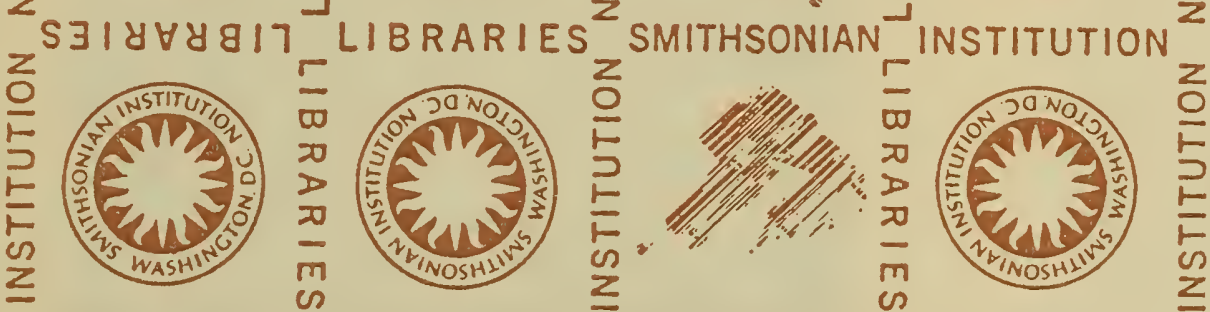

S SMITHSONIAN
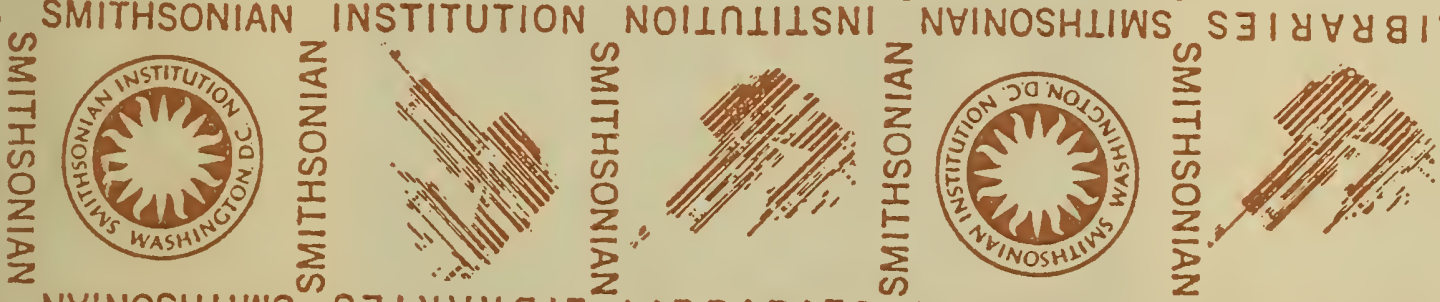

$z$
$\frac{1}{2}$
0
I
I
$\sum_{n}$
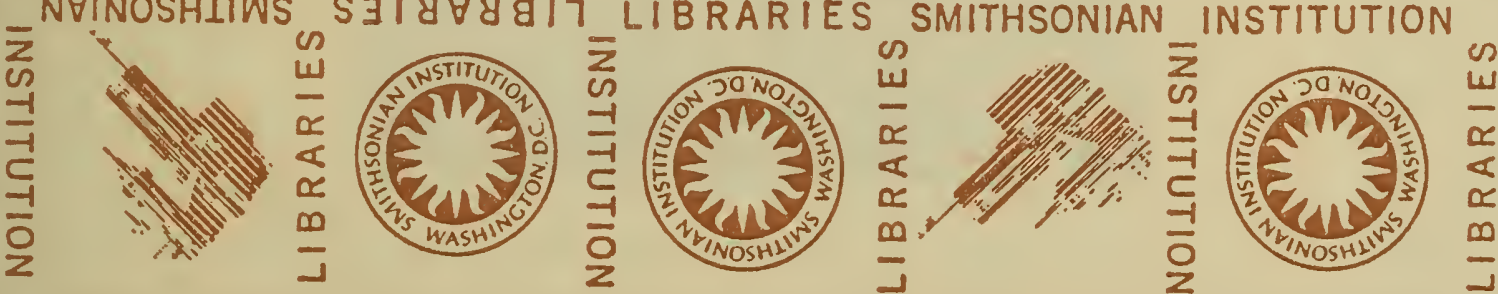

$S$ SMITHSONIAN INSTITUTION
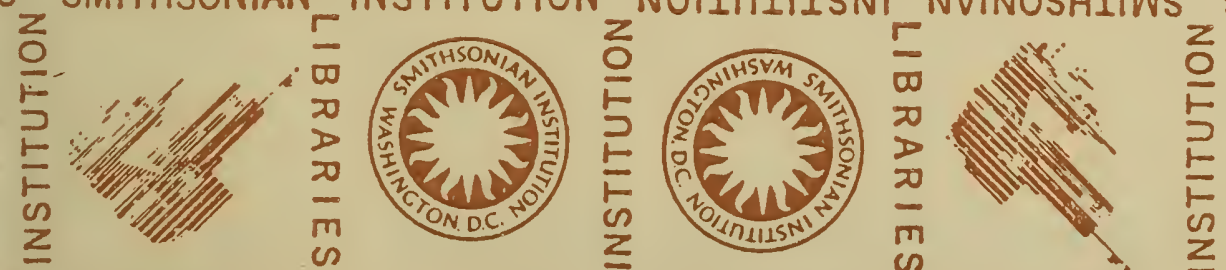

S $\exists \mid 8 \forall \forall 917$
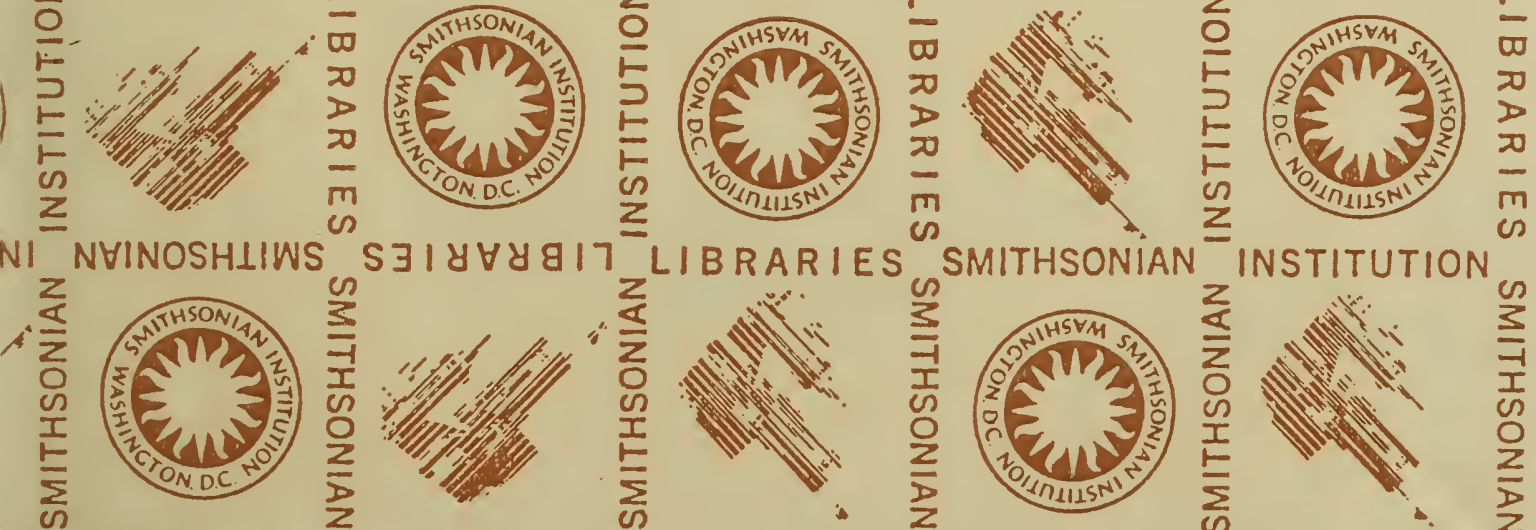

S SMITHSONIAN

INSTITUTION

NOIINLIISNI NVINOSHLIWS

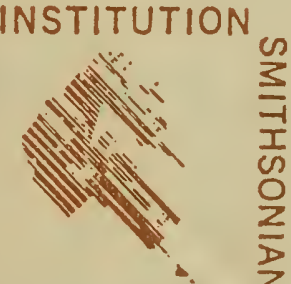



Tा

848

C26

CHM

AD, LEAFLET No. 100
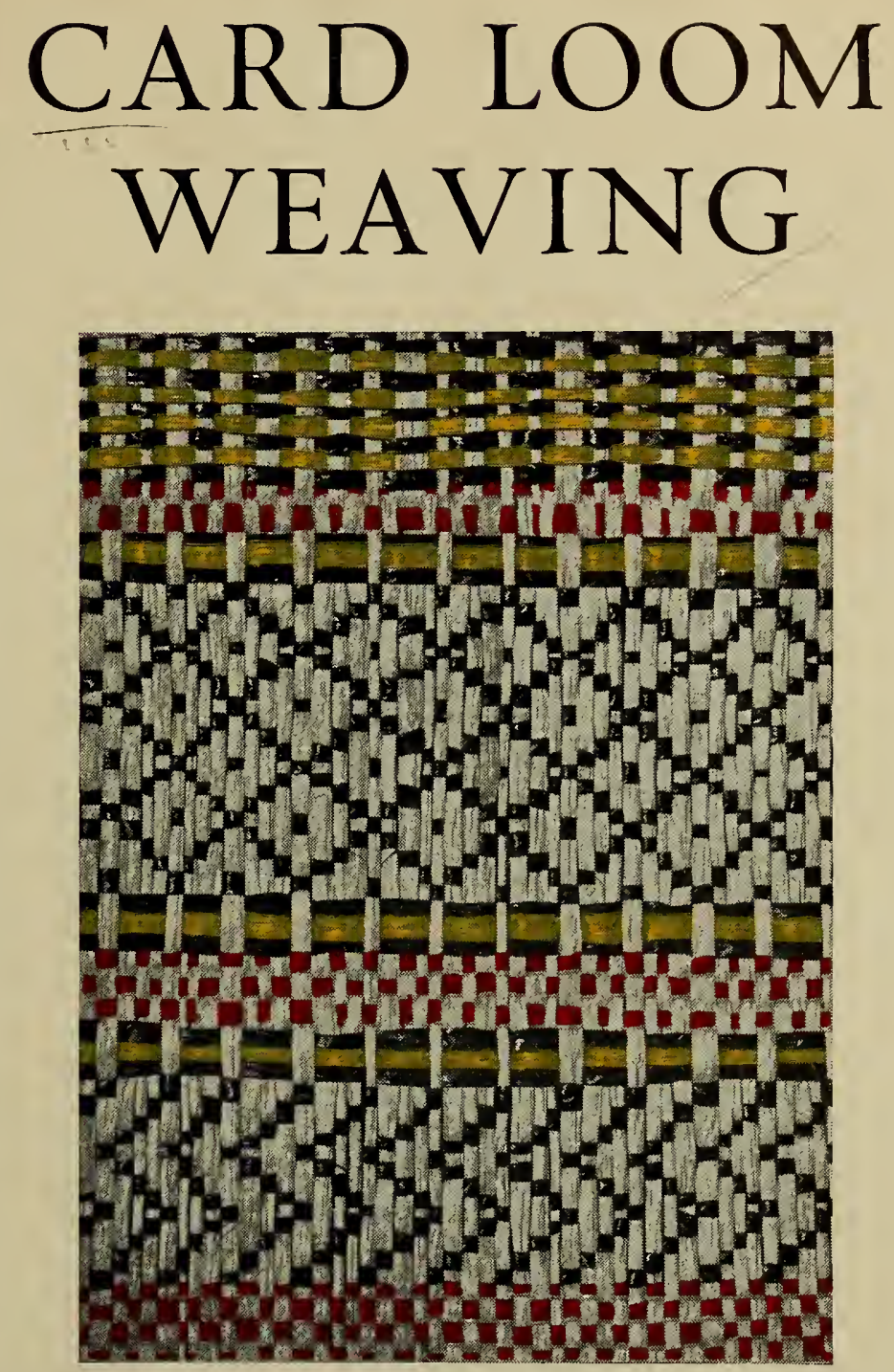

S A M P L R OF T WIL L WE A V I NG.

DRYAD HANDICRAFTS LEICESTER 


\section{CARD LOOM WEAVING}

The use of card looms forms an interesting introduction to the early stages of hand-weaving and with this simple apparatus it is possible to study the rudiments of design and use of colour in textiles and to produce an extensive range of articles which will be of great value in the infant and junior classes. It is suggested that from the beginning children should be provided with either coloured chalks or coloured paper and scissors with which to experiment in designing the simple striped effects to be introduced into the weavingbeginning with weft striping for the shaped cards, as in these the warp is covered and later for the rectangular cards striping in the warp and a combination of the two to form plaids. The card looms for finer material will be found to be very helpful to the student and senior scholar when working out a unit of pattern to be repeated to a required width when preparing to make a warp for a length of material to be woven on a larger loom. In this leaflet instructions are given for the use of various types of card looms. The cards are obtainable for work in graded stages - the simplest, requiring no tools, will therefore be found to be very suitable for the use of quite young children.

\section{BASKETRY FOUNDATIONS.}

These cards or Basketry Foundations, as they are named, serve to introduce the general principle of the use of the weft thread - the passing under and over and the binding together of the warp strands. In this case the card itself forms the warp, being cut with deep serrations at regular intervals leaving sections which are bound with raffia to form small trays, mats, bowls and baskets. To prepare a card for weaving it is necessary to stick paper to the centre portion of each side or as an alternative woven raffia fabric would be found to be more durable and could be sponged over when used if necessary. Some teachers will choose to prepare the edges for the final binding at this stage before the weaving is commenced. To do this either punch or pierce holes at even distances with the centre of the holes at about $\frac{1}{8}$ " from the edge of each section of the card. It is a matter of choice as to whether the holes are made before or after the article is woven. 
The weaving of the foundations for the making of trays and mats is a very simple process. Either raffia or cotton yarn may be used as the weft quite successfully, the thread being passed under and over the sections of the card in rotation with the fingers. For the making of bowls and baskets the best material to use is raffia, and when one or perhaps two colours are used in conjunction with the natural shade, introducing very simple striping, most attractive and useful articles can be woven. Before commencing to weave the foundations, bend each section of the card up from the base along the dotted line as shown in diag. 1 to form the required shape as in diag. 2. When it is necessary to introduce a new weft strand allow the end of the used strand to protrude on the under side of a mat foundation or on the outer side of a bowl and place the new strand into position for weaving - starting one or two sections back allowing the new end to protrude as before. Continue weaving and when work is completed carefully cut away all ends. After the final round of weaving darn the end of raffia away behind the weaving for the full depth of a section of the card. To finish off the article work a row of overcast stitches into the holes prepared at the edges of the sections from left to right (diag. 3) and when reaching the starting point return in the opposite direction working a second row of stitches into the same holes. This binding thread must be finished off very carefully, the final end being darned under the overcasting for a short distance and then cut away.

\section{FIRST USE OF SERRATED CARD LOOMS.}

As a preliminary exercise the method followed in paper mat weaving may be used on the straight serrated card looms measuring $6 " 1$ × 6" for the making of doll's housc carpets, etc. In this way the difficulty of keeping a straight edge is -avoided. The warp of cotton yarn is wound on to the card as shown in diag. 6-the short ends at the commencement and at the end being stuck to the card during use. A short length of cotton is used for each row, being cut sufficiently long to protrude beyond the warp threads on both sides of the card from $2^{\prime \prime}$ to $3^{\prime \prime}$ to form a fringe. A needle, with turned up point, is used for the weaving, every row being 


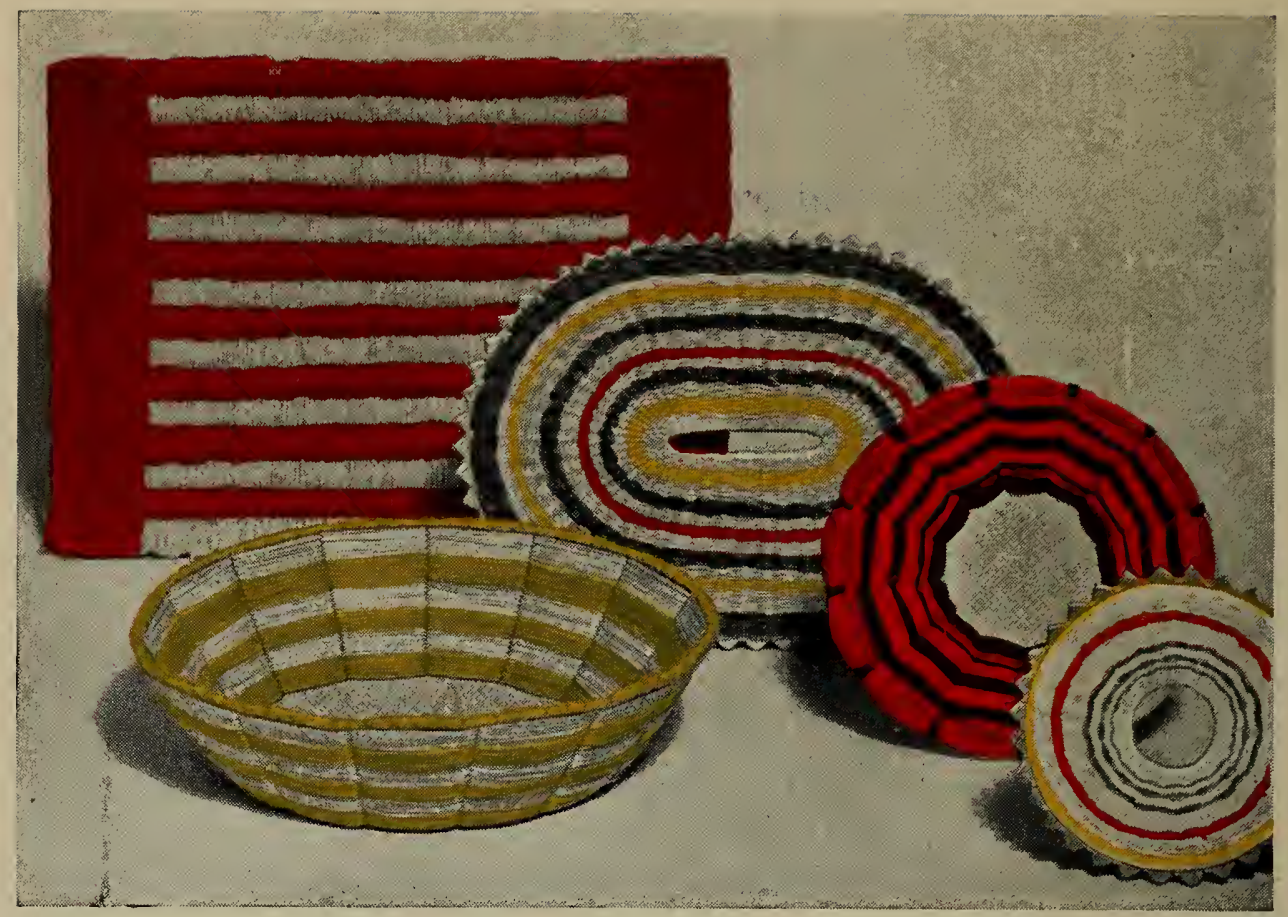

ARTICLES MADE ON BASKETRY FOUNDATIONS A ND SERRATED CARDS.

woven from right to left, in the first row picking up alternate strands, in the next picking up the strands covered by the previous row and pressing each row down into position with the point of the needle so that the weft threads are close together. When the weaving is completed a row of overcasting or hemstitching should be worked round the ends forming the fringe and into the weaving. To remove the work from the card ease the loops of warp over the serrations and if necessary weave a further row or two into the loops to complete.

\section{SHAPED SERRATED LOOMS.}

Of the several shapes supplied in the serrated card looms the round are the easiest to handle. The introduction of the use of the warp strands is made at this stage and it is quite possible for children to undertake the process of winding the warp without any difficulty. This is done directly on to the card as illustrated in diag. 4-passing the warp yarn through the centre hole and round the card, allowing one thread to lie in each serration. So that the first end will stay in position during the winding process tie it temporarily to 
the second warp. All joins should be made on the under side of the card away from the edge. When the winding is completed untie the first end from the second warp and tie it securely to the last end. The best knot to use for the joins is a reef knot as it lies quite flat and can be easily hidden with the weft thread. Raffia, medium twine or fine cotton yarn are suitable threads for warp with either raffia or 4-ply wool as weft.

A needle with turned up point is used to carry a long weft thread, being passed under and over one strand throughout. Each successive row of weaving must be pressed into position with the point of the needle. At this stage a natural coloured raffia or wool should be used with only one or two colours to form simple striped effects. Begin weaving at the centre of the card with the colour used for the warp. If the weft is of raffia, for the first inch or so of weaving it is well to use quite narrow strands, the thickness being gradually increased as the weaving progresses. When introducing a new length of weft thread either allow the new and old strands to overlap for a short distance or join the two ends together with a reef knot and arrange for it to lie under a warp strand. Press the rows of weft well together but do not pull the thread tightly. Both sides must be woven, the card remaining inside the weaving to form a firm foundation for the mat. The serrated edge may afterwards be enamelled if desired. When preparing the tea cosy attach a small ring as shown in diag. 5 on each side of the card. Do not tie the thread which is used to hold the rings in position too tightly or it will be difficult to pass the warp under the rings when setting up the card. If a length of twine is tied to the ring on one side, passed over the bottom serration and tied to the ring on the opposite side of the card, it will help to hold the rings in position while winding the warp. It is of course removed when the warp reaches this -point. Commence by tying the warp, which may be either of raffia or medium twine, to one of the rings, leaving an end about an inch in length-which is afterwards placed along the first warp strand and covered with the weaving. Pass the warp thread over the first serration and through the ring on the other side of the card. Return in the opposite direction over the next serration and through the first ring 


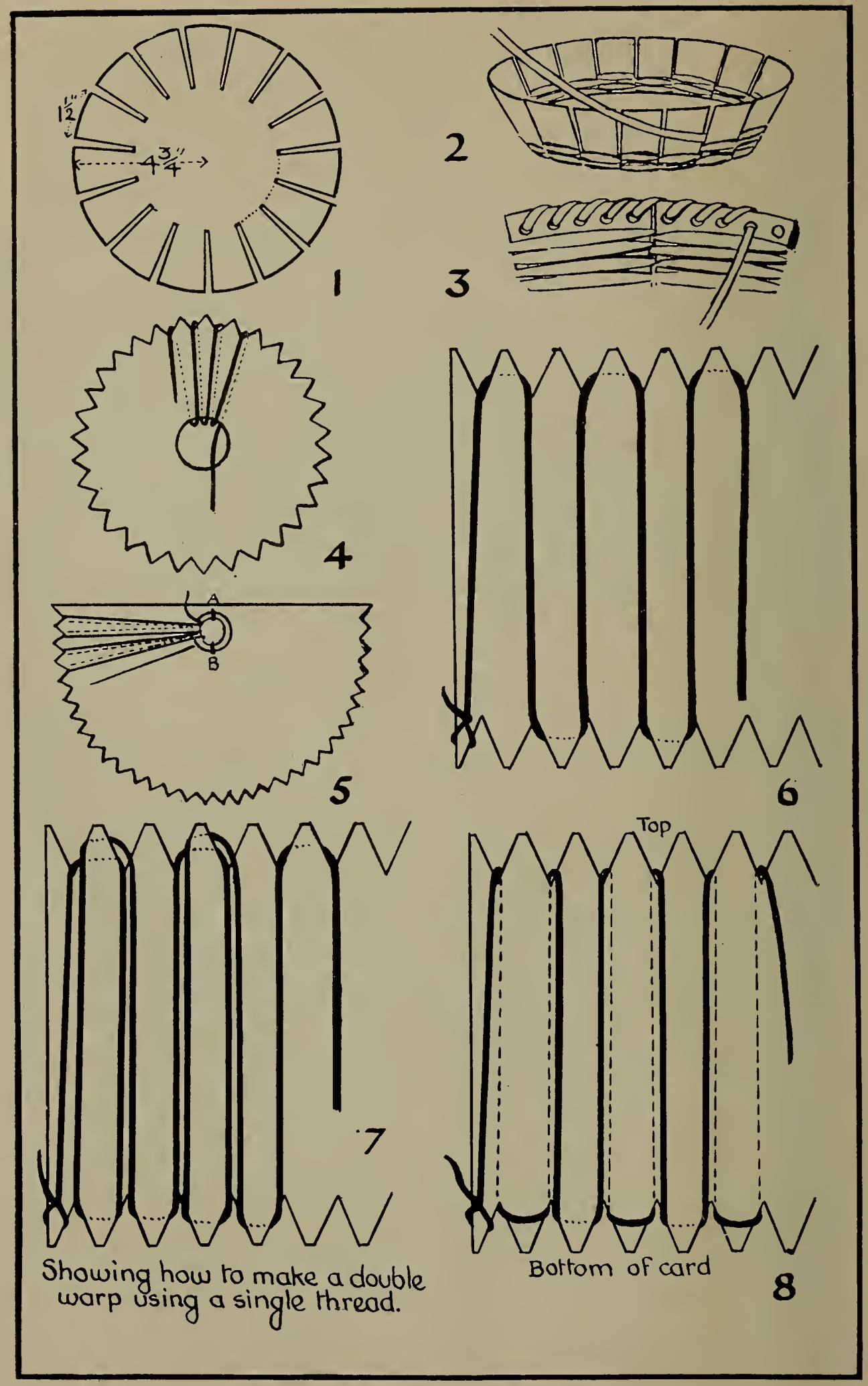


again-repeating this process until all the serrations are filledfinally tie the end warp strand to the ring as at the beginning. The method of weaving has already been described-commence close to the ring leaving a short end unwoven, and follow the semi-circular shape of the card from right to left-return from left to right, passing the needle under the threads that were covered in the previous row-do not pull the weft thread tightly or it will tend to draw the warp out of shape. It will be found helpful if a line is drawn on the card as the end warp threads lie before weaving is commenced and to turn each row carefully at this point. The short end left at the beginning is darned in with the second row. As the warp strands become filled they must be omitted and the weaving continued in a series of steps until the card is filled on one side. The second side is then woven in a similar manner with corresponding striping. When completed crease and tear the card and slip it away from the weaving. After it has been removed it may be necessary to weave three or four extra rows into the space left by the card.

Blanket stitch round the exposed portions of the two rings and finish the article for which purpose it is required. If tea or egg cosy, make lining of felt, and if a bag linen or casement cloth might be used as alternatives. Suggestions for linings and the making of handles are given later in this leaflet on pages 12,13 and 14 .

\section{SERRATED CARD LOOMS.}

With these straight serrated card looms it is possible by the arrangement of the warp threads to produce plain or tapestry weaving. When the warp is wound from a single thread with one in each serration the weft threads will cover the warp and produce what is known as a tapestry weave. But when the warp is double, see diag. 7, and the weft threads are passed - under and over alternate single threads an equal amount of warp and weft will be seen and the resulting material, giving the appearance of a square mesh, is what is termed plain or "tabby" weaving. With this weave it is possible to introduce additional interest into the work by using two or three colours in striped effect in the warp, and also by crossing the warp with the weft in a similar striping to form simple plaid patterns. 
The tapestry weave is very effective when making tea cosies, pochettes, etc., where a thick material is desirable and the tabby weave is more suitable for the small scarves, table runners and dish cloths which can be made on these simple card looms.

The first method of winding using a single warp thread on one side of the card is shown in diag. 6. Either the cotton weaving yarn, 4-ply wool or raffia may be used in this way for the making of such articles as doll's house mats, purses, small pochettes, etc., using either material for warp and weft respectively, producing a tapestry effect. The second method shown in diag. 7 describes how to mount a double warp from a single thread on one side of the card. The warp being more closely set in this way, plain or tabby weaving can be produced with either 4-ply wool, raffia or cotton weaving yarn as warp and weft by weaving under and over one thread. The white medium cotton yarn will be found suitable for the making of dish cloths in this way. The third method illustrated in diag. 8 shows how both sides of a card are wound from either a single or double thread according to the texture required.

Commence by tying the warp thread to the end serration on the left hand side of the card, leaving a short end; continue across the card making any joins, unless otherwise stated, at a short distance from the serrations. When the required width has been wound tie the end of the warp to the last serration, leaving a short end as at the commencement. It will be found helpful if these ends are stuck to the card while weaving.

The weaving presents a difficulty to the beginner-one that can only be overcome with careful practice. This difficulty is the keeping of straight edges and the preventing of the drawing in or "waisting" of the weaving. As with the cosy and bag cards, it will be found helpful to draw a line on the card-on these straight cards parallel with the second warp strand from each edge, and to turn the weft leaving even loops at this point. The thicker the material used for warp and weft the easier it will be to weave evenly. A needle is used for the weaving, as on all card looms, picking up alternate threads from right to left and returning from left to right for the next 


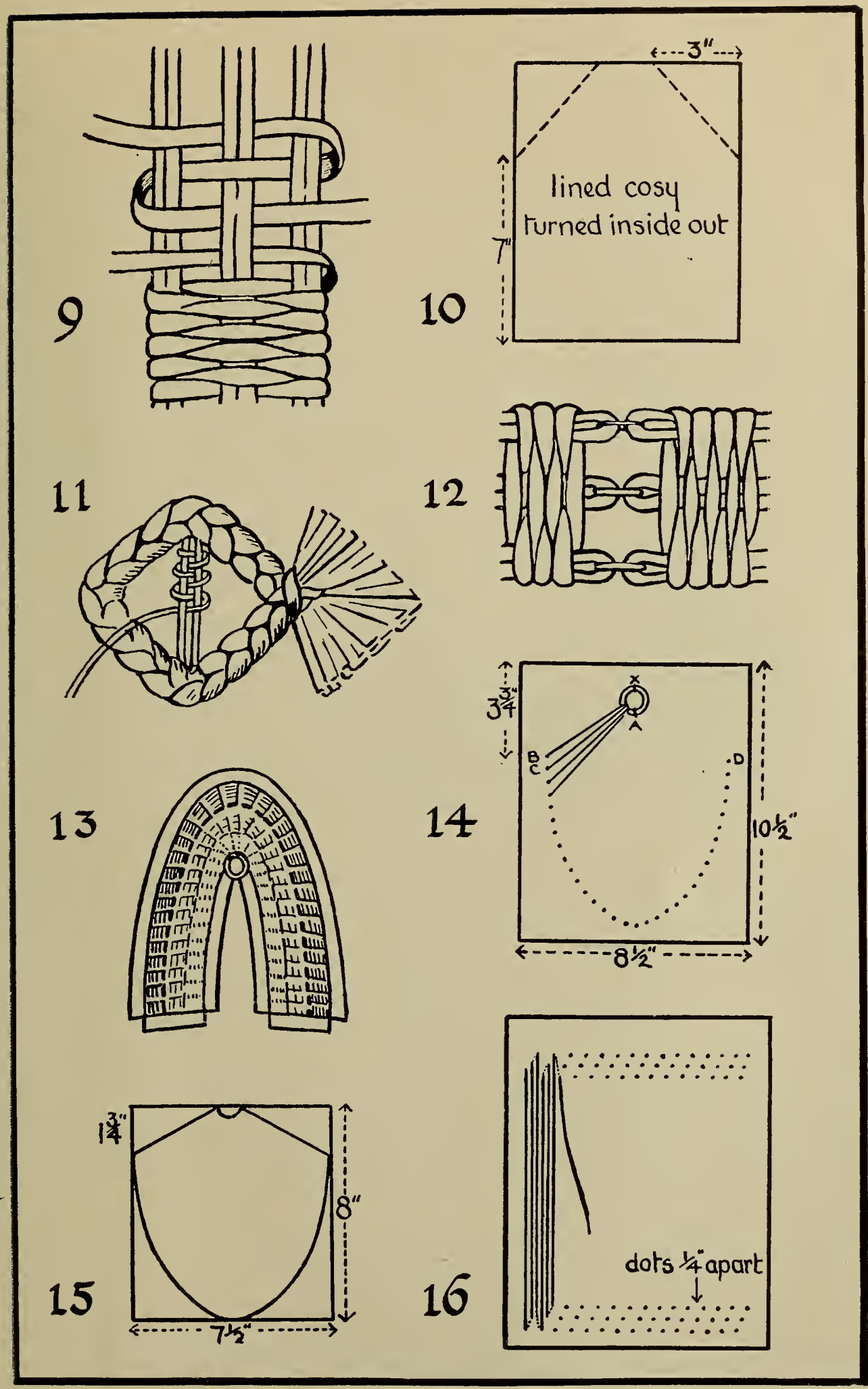


row, as in darning, picking up the threads that were covered with the weft in the previous row. A short end is left unwoven at the commencement and then darned back into the second row. The point of the needle is used to press the rows of weaving together.

In tabby weaving when it is necessary to introduce a new thread of the same colour allow the old and new strands to overlap for a short distance as in diag. 9, but when new colours are to be used in striping, cut the colour which is to be dropped, leaving an end unwoven, and fold it round the end warp strand and darn into the same row. Then weave the next row introducing the new colour from the opposite side. The joining of weft threads in tapestry weaving on card looms must be done very securely as the material is somewhat looser than in tabby weaving. The ends of the old and new strands may either overlap or be tied with a reef knot arranged to lie under a warp thread. The knots will be covered by the successive rows of weaving.

With care these cards may be used several times. To remove weaving simply lift loops from the points of the card and where the weaving is continued for the full extent of the loom an extra row or two must be darned into the space left by the card. If a length of warp has been left unwoven at one end of the card to be used after as a fringe, either hemstitch along the edge of the weaving round two warp strands and down two rows of weft and then cut across the warp trimming the fringe, or cut through the loops first and knot together, two, three, or four warp strands slipping the knots close to the first row of weaving and then trim the fringe to the required length. It is possible to weave a child's scarf from two lengths setting up the card each time in the manner of diag. 8 and weaving both sides of the card using 4-ply wool for a double warp. The same wool is also used for weft in a tabby weave.

The coffee pot cosy illustrated on page 12 can be made from the measurements given in diag. 10 .

In diag. 11 a plaited raffia buckle for a dress belt is illustrated. The belt, illustrated on page 13 , is made by weaving two narrow strips on a serrated loom and joining them together 


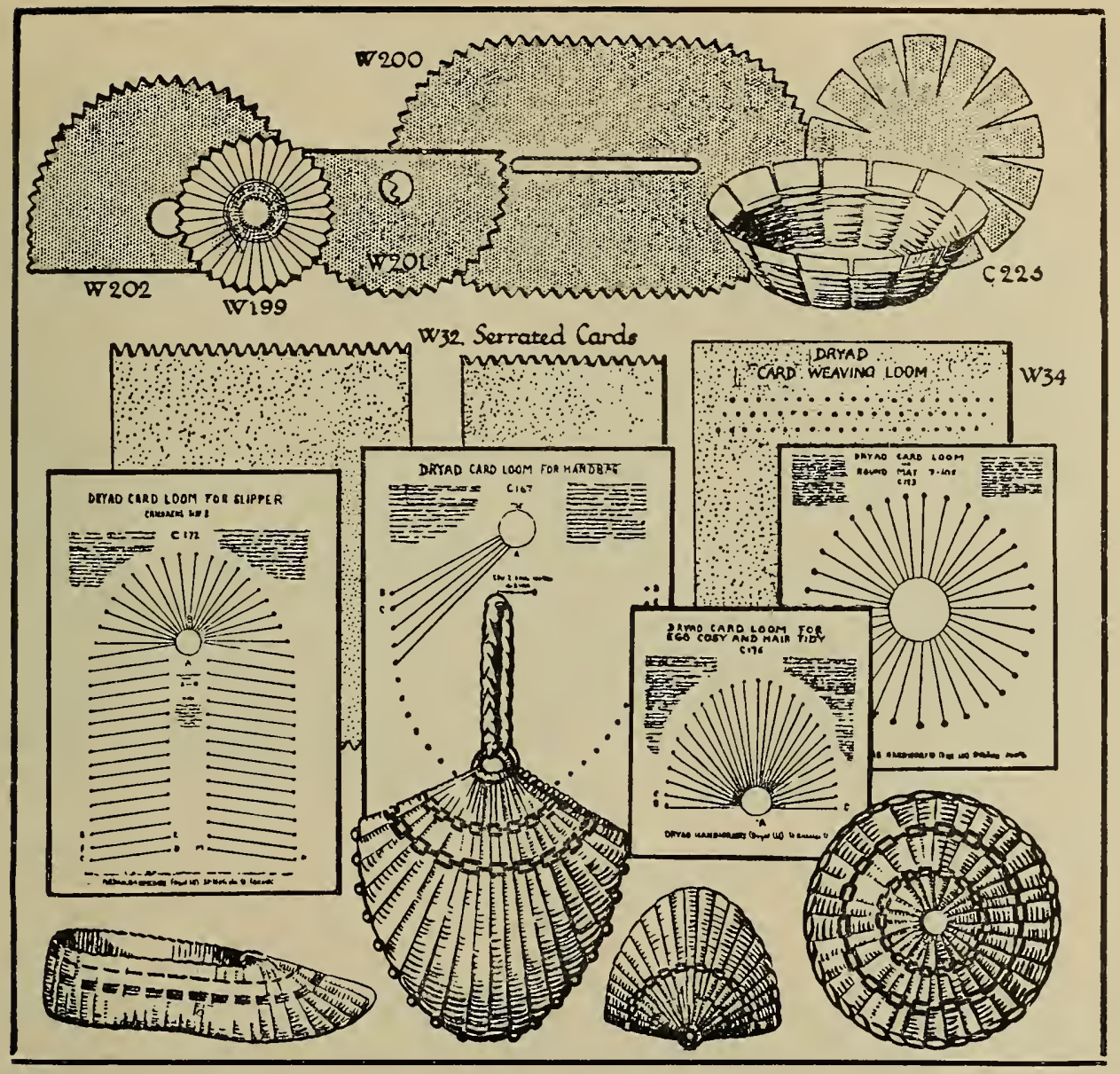

C A R D F OR WE A V I N G.

in the centre by tying the opposite loops in the two pieces of weaving and afterwards covering them with a few more rows of weaving, as shown in diag. 12.

\section{SHAPED LOOMS.}

These card looms are printed with a dotted outline of the shape of the article to be woven and are supplied for the making of mats, bags, cosies, slippers and berets. The method of arranging the warp strands is explained on each card. Either raffia, medium twine or 4-ply wool form suitable warps for most of the cards, though in the case of the berets it is best to use 4-ply wool only for warp and weft, and when making slippers use medium twine for warp with either raffia, 4-ply wool or rug wool, after about $\frac{1}{2}$ " has been woven in 4-ply wool, as weft. Make all joins in the warp at a short distance away from the holes. 


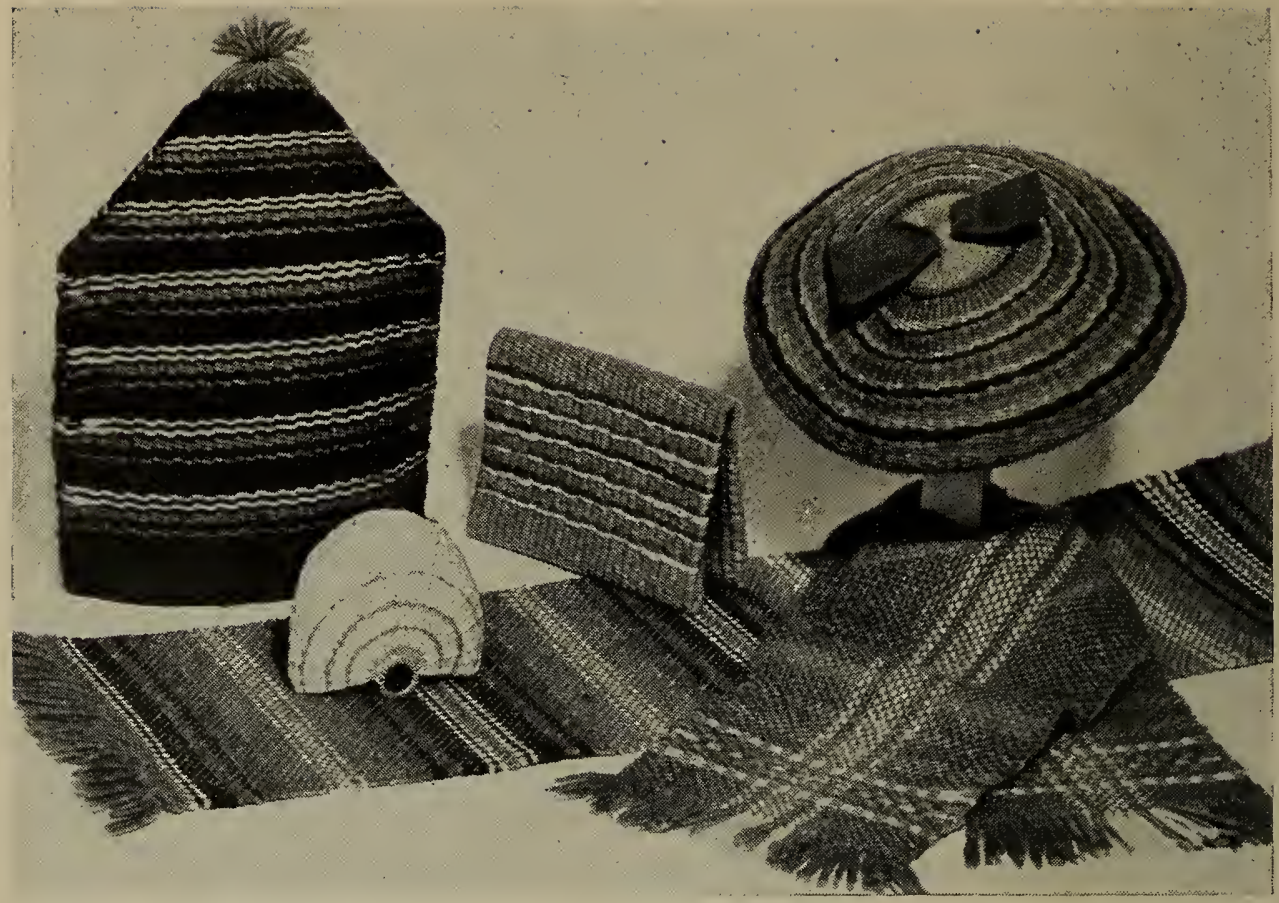

ARTICLES MADE ON SHAPED AND RECTANGULAR C A R D L O O MS.

The weaving is carried out in a similar manner to that already described, omitting warp strands in steps where necessary and continuing the weaving on the remaining strands to obtain the desired shape. When the weaving is completed the card is torn away along the lines of holes, the rings released and the card removed.

The making up and lining of the various articles will naturally vary somewhat according to the shape of the weaving. For bags, cosies, pochettes, etc., the material most suitable for the purpose of lining is felt. (Do not use a damp cloth when pressing felt or it will be inclined to shrink.) In some cases, however, coloured linen will be found to be more suitable.

Exposed portions of the rings should be covered with blanket stitches in the material used for the weft.

In the finishing of slippers-felt is the best material to use. Place the weaving on the felt and tack together very carefully with long zig-zag stitches. The surplus felt is then cut away to within $\frac{3}{8}$ " of the outer edges of the slipper and at the straight 


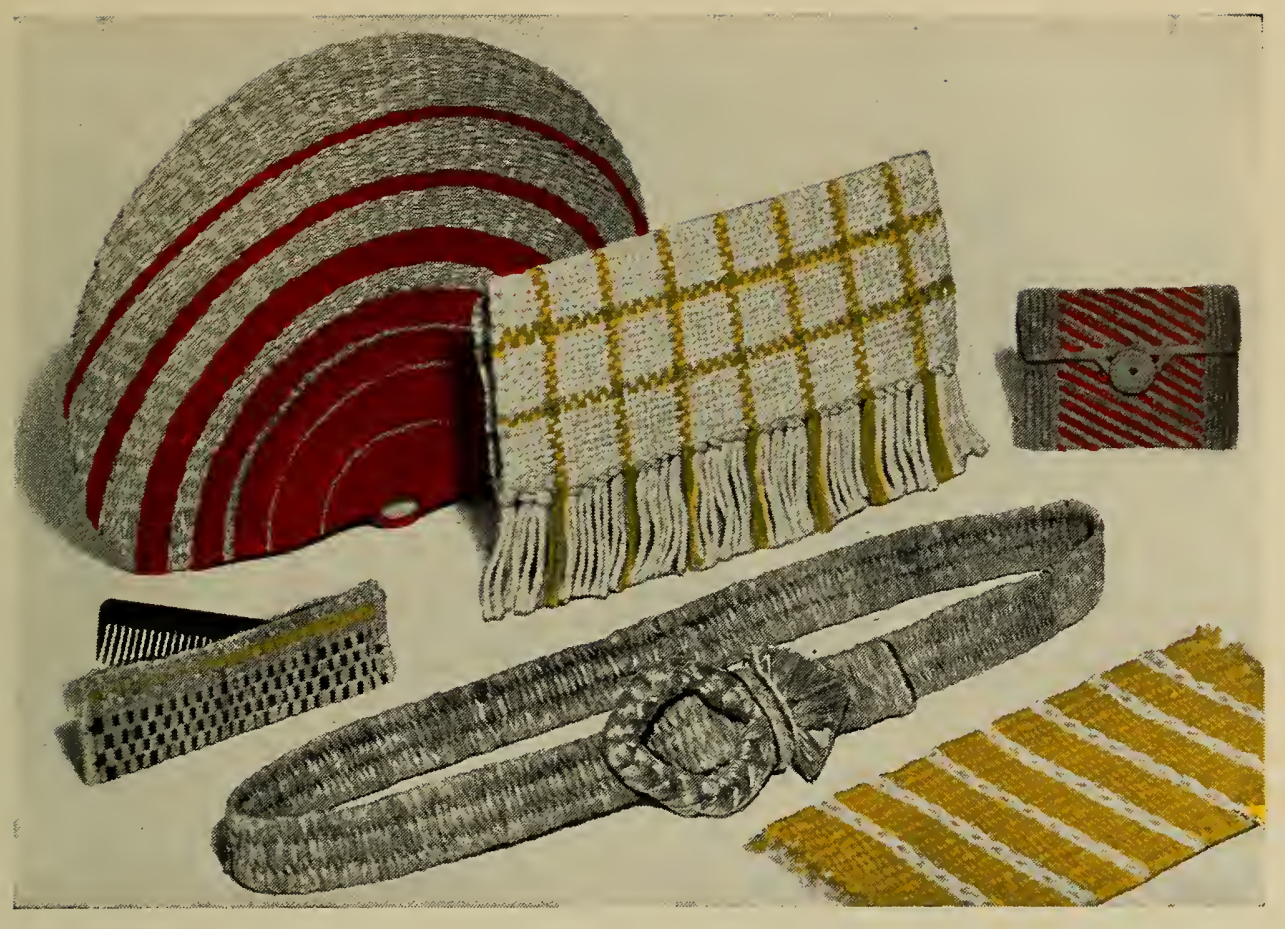

ARTICLES MADE ON SHAPED A ND RECTANGULAR C A R D LOOMS.

ends (shown in diag. 13), also to within $\frac{3}{8}$ " of the inner edges as far as the ring following close to the outline of the ring. The felt protruding beyond the weaving is used as binding. First place the straight edges together and sew through the felt close to the outer edges of the weaving, then fold back the straight edges of the felt and tack into position. Attach with a row of double running stitches near the cut edge of the felt, through the binding, woven material and lining. Next gather the felt very slightly, close to the weaving, for a short distance at the toe and at the heel and then join the binding, by oversewing, at the straight ends. Fold back the binding at the top and bottom of the upper and sew into position with double-running stitches. Blanket-stitch round the upper portion of the ring and join to the lining-cover the remainder of the ring with a small felt bow to finish.

Leather soles, with lining attached, may be obtained in sizes to correspond with the various cards supplied. To attach upper to sole first catch together, temporarily, at the centre heel and 


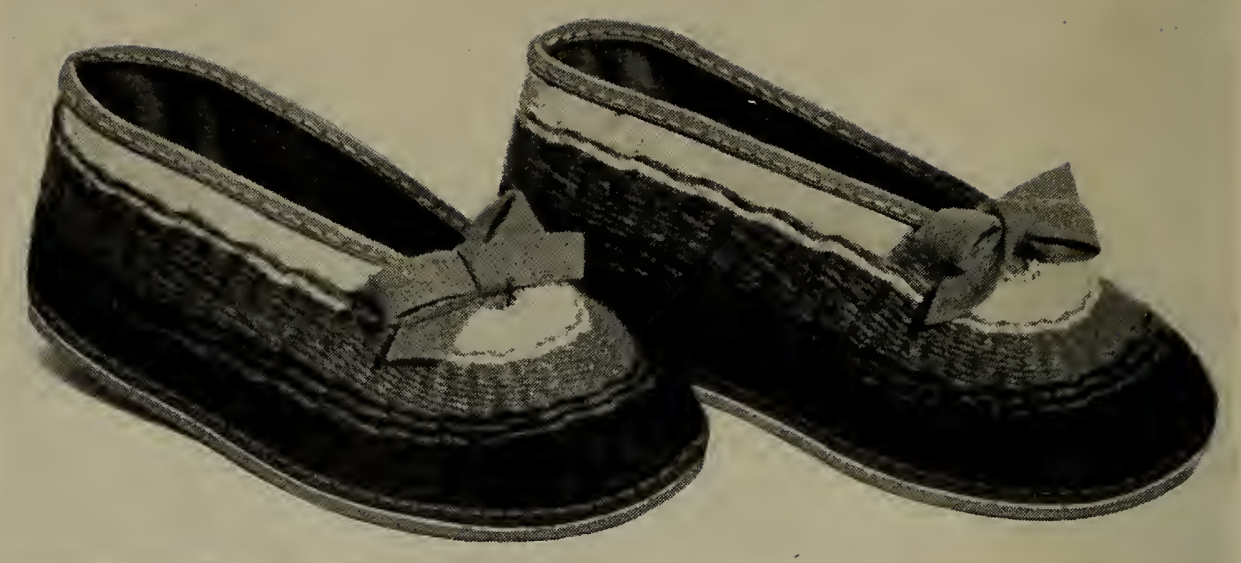

SLIPPERS MADE ON A SHAPED CARD LOOM.

at the centre toe, also at intervals between these points. Sew the upper to the sole as shown in diagram 17, using two needles. Begin at the heel and work one side towards the toe, and then begin again at the heel and work along the opposite side to the toe.

In the case of a beret, when the weaving is removed from the card a strip of felt approximately $1^{\prime \prime} \times 3^{\prime \prime}$ in the colour used for the warp should be inserted under the warp strands, which cannot be drawn together with the weft thread in the centre of the crown at the commencement. This will also add style to the beret. A circle of warp sateen sewn inside the top of the weaving will form a suitable lining and will also give added strength and help to retain the shape of the article.

Handles will be required for several of the bag shapes-for these either weave narrow strips in the same material or make a firm plait or twisted cord of a suitable length and thickness. A three or four-plait could be used with several strands in each section. When complete either knot or bind the ends to secure. When making a twisted cord handle take the necessary number of threads to give the required thickness in either wool or raffia and knot the strands at each end. Assistance is required-one person manipulating each end of the twist. A pencil is placed through the strands at each end and holding them taut each person turns her pencil to the right until the twist is very tight. The worker then holds the twist in the centre and hands her end to the assistant, still retaining the tension of the 


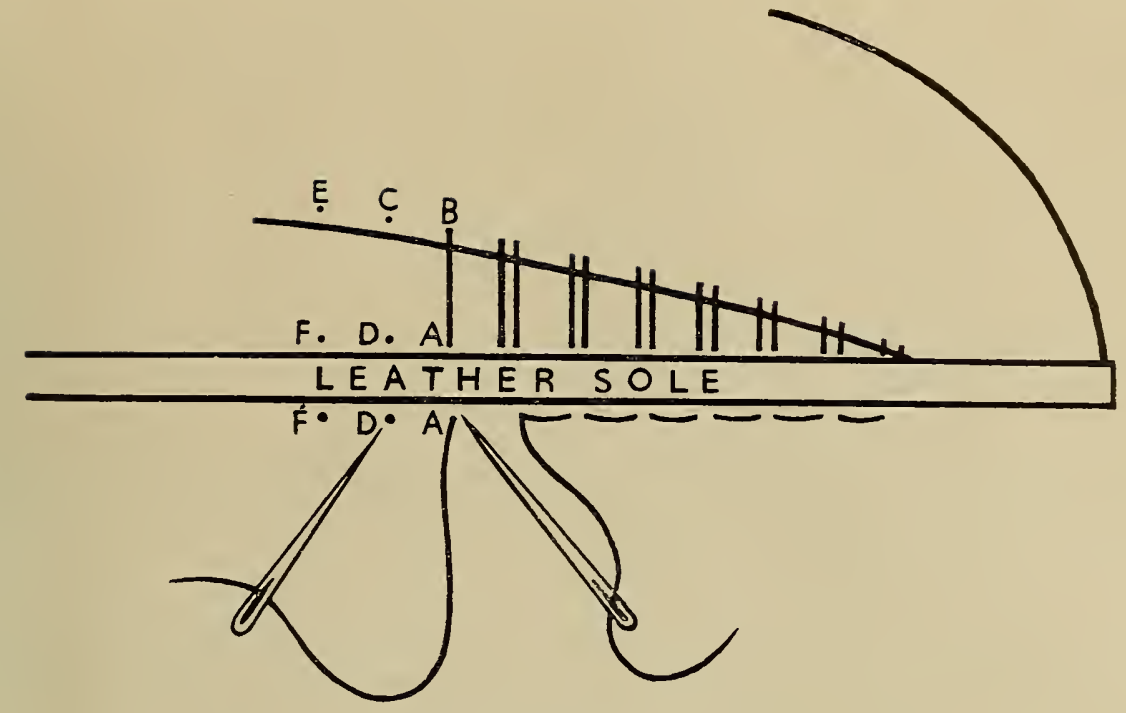

D I A G R A M 17.

Ist needle-thread up through $\mathrm{A}$ in the sole, down through $\mathrm{B}$ and up through $\mathrm{C}$ in the upper; down through $\mathrm{D}$ in the sole.

2nd needle-thread up through $\mathrm{D}$ in the sole; down through $\mathrm{C}$ and up through $\mathrm{E}$ in the upper; down through $\mathrm{F}$ in the sole. Continue as described above using the needles alternately.

twist. The ends are held securely and the centre of the twist released. It will then form itself into a cord. Any puckers must be smoothed away and the ends knotted together.

To attach, each end of the handle may be placed through a ring and folded back onto itself, bound and stitched to secure -or placed straight through the rings and stitched into position and the fringed ends trimmed to neaten.

When a large portion of any article is without striping introduced into the weaving it is possible to embroider simple stitches arranged in a definite order to give additional interest to the design, loop stitches being very suitable for the purpose.

In diag. 14 the method of warping a bag card is shown and in diag. 15 the shape and size of the lining for the particular bag is given as a guide.

\section{CARD WEAVING LOOMS.}

These rectangular card looms are printed with a series of dots along the top and bottom edges in such a way as to allow the use of finer threads for tabby weaving without weakening the card as shown in diag. 16. 
Instructions for the arrangement of the threads will be found printed on the cards, joins in the warp being made with reef knots by the holes at either the top or bottom edge. It is now possible to experiment with finer threads in the designing of fabrics to be woven on the larger looms-always bearing in mind that the small pieces of material woven on the card looms may be utilised either as sampler specimens or for some useful or decorative purpose.

The most suitable materials for use on these cards are 3-ply wool and fine cotton weaving yarn, both of which are obtainable in a good range of colours.

The method of weaving on these cards has already been described in the paragraph on the use of the straight serrated cards (p. 7). When the weaving is completed, neatly oversew or hem-stitch along the two ends and then cut the loops at the back of the card-trim the ends to neaten before lining and making up.

This leaflet, one of a series covering practically every branch of craftwork, is published by Dryad Handicrafts, who also supply materials and tools for all the basic crafts. Particulars will gladly be forwarded on application.

\section{R Y D H A N D I C R A F T}

\section{SAIN T NICHOLAS ST., LEICESTER}

London Showroom: 22 Bloomsbury Street, W.C.1. 
SMITHSONIAN INSTITUTION LIBRARIES

chm $\pi 848 . C 26$

Card loom weaving 